\section{Diaphragm-based extrinsic Fabry-Perot interferometric optical fiber sensor for acoustic wave detection under high background pressure}

\author{
Ming Han \\ Xingwei Wang \\ Juncheng $\mathrm{Xu}$ \\ Kristie L. Cooper \\ Anbo Wang \\ Virginia Polytechnic Institute and State \\ University \\ Center for Photonics Technology \\ Blacksburg, Virginia 24061-0287
}

\begin{abstract}
A new structure for diaphragm-based extrinsic Fabry-Perot interferometric (EFPI) optical fiber sensors is presented. This structure introduces a through hole in a conventional diaphragm-based EFPI sensor and significantly reduces the effect of operating point drift due to the background pressure and fluctuations. This structure also potentially has high temperature stability. (c) 2005 Society of Photo-Optical Instrumentation Engineers. [DOI: 10.1117/1.1924689]
\end{abstract}

Subject terms: fiber optic sensors; Fabry-Perot interferometers.

Paper L040816 received Nov. 1, 2004; revised manuscript received Mar. 24, 2005; accepted for publication Apr. 7, 2005; appeared online Apr. 11, 2005; published online Jun. 9, 2005.

\section{Introduction}

Currently, fiber optic extrinsic Fabry-Perot interferometric (EFPI) sensors have been studied extensively for acoustic signal detection due to their inherent advantages including small size, light weight, high sensitivity, high frequency response, electrical nonconductivity, immunity to EMI noise, and single-point measurement. ${ }^{1}$ Among them is a diaphragm-based EFPI sensor comprised of a fused silica diaphragm and a single-mode fiber (SMF) end., ${ }^{2,3}$ The vibration of the diaphragm caused by acoustic waves operates the interferometer in the linear range of one of its interference fringes. The sensors are designed to be sensitive to pressure changes in order to measure acoustic waves. However, small fluctuations of background pressure could change the operating point of the sensor in an unpredictable way. Several approaches, such as multiwavelength interrogation ${ }^{4,5}$ or spectral interrogation ${ }^{5}$ and Q-point control using a bandpass filter, ${ }^{3}$ have been employed to compensate the operating point drift, while the price is a significant increase in system complexity and cost. In this letter, we present a new structure for diaphragm-based EFPI optical fiber sensors to eliminate operating point dependence on background pressure fluctuations.

\section{Principle of Operation}

The sensor structure is shown in the inset of Fig. 1. A SMF zirconia ferrule with outer diameter $D=2.5 \mathrm{~mm}$ is inserted into the ferrule sleeve, leaving a small distance $L$ $=0.2 \mathrm{~mm}$ between the ends of the ferrule and the sleeve. A fused silica diaphragm of thickness $170 \mu \mathrm{m}$ is bonded on the end of the sleeve. The sleeve has a 0.3-mm-wide slot initially designed for the easy insertion of the ferrule. In this structure, the slot, together with the diaphragm and ferrule, forms a $0.2-\times 0.3-\mathrm{mm}$ through hole connecting the air cavity with the outside environment. The cleaved lead-in fiber is then inserted into the ferrule, forming an air gap $d$ between the fiber end and the inside end of the diaphragm. The other side of the diaphragm is roughened by sandpaper to eliminate undesirable reflections. The air gap can be properly adjusted using a white light system. The fiber, ferrule, ferrule sleeve, and diaphragm are all bonded together by epoxy.

Since the "through hole" area $S_{h}$ is much smaller than that of the diaphragm $S_{d}$, the acoustic energy diffracted into the air cavity through the hole is negligible. Suppose the acoustic energy diffracted into the air cavity is proportional to the area of the hole $S_{h}$. In our case, $S_{h}$ $=0.06 \mathrm{~mm}^{2}, S_{d}=\pi(D / 2)^{2}=4.91 \mathrm{~mm}^{2}, S_{h} / S_{d}=0.012$. The acoustic energy diffracted into the cavity is only $1.2 \%$ of the energy applied on the diaphragm. The sensitivity to acoustic waves does not decrease much compared to a sensor without a through hole. However, for background pressure, which is characterized as a static pressure with slow fluctuations, the pressure inside and outside the air cavity will be quickly balanced by the through hole and will not change the air gap. Thus the sensor is expected to be insensitive to the background pressure while maintaining its sensitivity to the acoustic waves. In addition, such through structure offers potential temperature stability, because it eliminates the effect of the trapped air expansion induced pressure, which is an issue in conventional hermetic sensors. Also, when an epoxy-free bonding method, such as laser bonding, is used, the sensor can survive hightemperature environments. ${ }^{6}$

\section{Experimental Results}

A static pressure test was first performed. The experimental setup is shown in Fig. 1. The optical light from a highresolution swept laser interrogator (HR-SLI) (Model V5.1, Micron Optics, Inc.) was coupled into the sensor through a 3-dB SMF coupler. The reflected signal from the sensor was collected by the same coupler and measured by the detector built in the HR-SLI. The sensor was sealed in a chamber connected to a high-accuracy pressure controller. Figure 2 shows the air gap of the sensor changes $12 \mathrm{~nm}$ when the pressure in the chamber increases from 20 to 80 psi. If we define the linear operation range of the sensor as the air gap change that causes a phase change of $40 \%$ of a full fringe period, then the linear range of the sensor at operating wavelength $1550 \mathrm{~nm}$ is $228 \mathrm{~nm}$. The air gap change due to a pressure change from 20 to 80 psi is only $5.25 \%$ of the linear operation range, which is negligible in most practical applications. Also, this can be further re- 


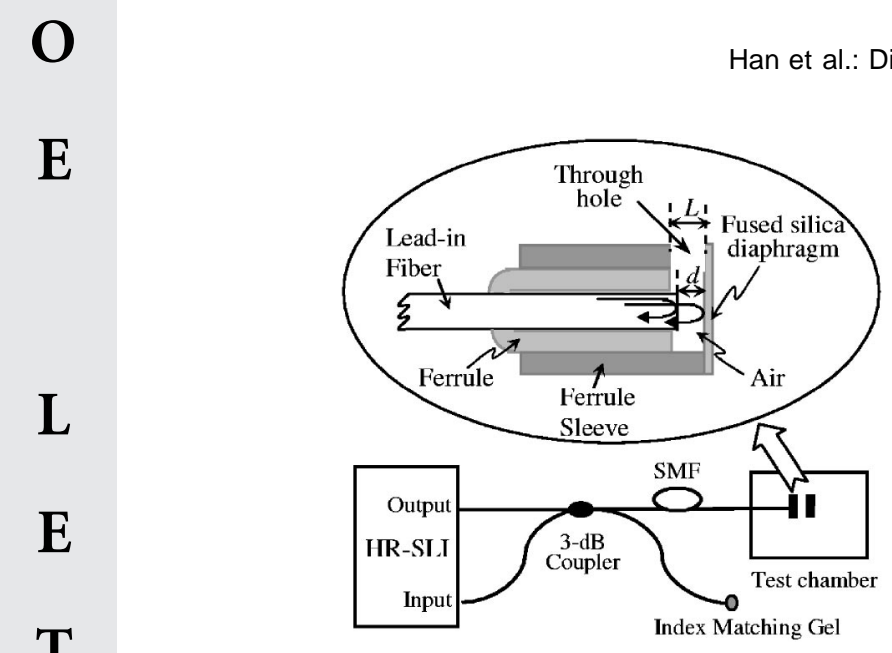

Fig. 1 Experimental setup for sensor test. The inset shows the structure of the sensor head.

duced by choosing a fused silica ferrule and sleeve, which are of the same material as the fiber, and using epoxy-free bonding.

The sensor was then applied to the acoustic wave test system. The setup is the same as Fig. 1 except that a tunable laser was used as the light source and a high-speed InGaAs detector together with an electrical current amplifier was used to transfer the optical signals from the sensor into electrical ones. A digital oscilloscope was used to display and store the acoustic signal captured by the sensor. An ultrasonic air transducer was sealed into the same chamber to apply acoustic waves to the sensor. Meanwhile the background pressure was controlled by the pressure controller. The wavelength of the laser was tuned to 1546 $\mathrm{nm}$ and maintained there during the test. Figure 3(a) shows the $46-\mathrm{kHz}$ voltage signal applied to the transducer. The waveform should be the same as the acoustic signal. Figure 3(b) shows the sensor signal displayed on the oscilloscope, which indicates the sensor is capable of faithfully detecting the acoustic signals. The experiment was repeated at different background pressures up to $80 \mathrm{psi}$. In all cases the sensor worked well with no need to adjust the operating point.

For a diaphragm-based sensor without a through hole, the sensitivity of the diaphragm is given by $\delta=2.524$

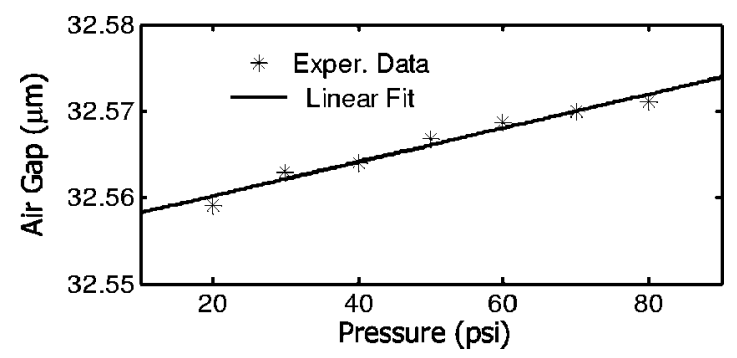

Fig. 2 Static pressure test: air gap change of the sensor versus pressure applied.

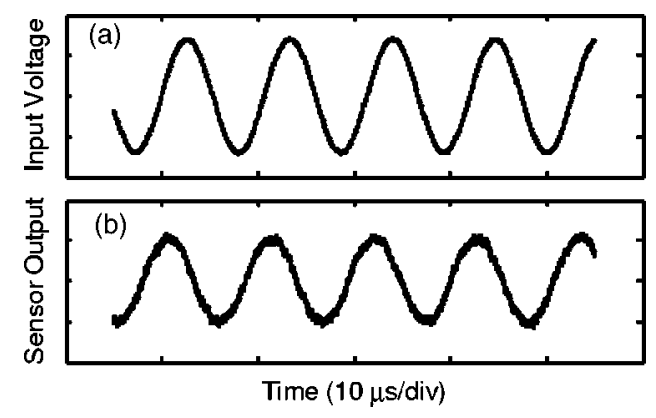

Fig. 3 Acoustic wave test at background pressure: $P=40$ psi. (a) Signal applied to the acoustic transducer. (b) Signal from the sensor.

$\times 10^{-6} a^{4} / h^{3}[\mathrm{~nm} / \mathrm{kPa}]{ }^{3}$ where $a$ is the diaphragm radius and $h$ is the diaphragm thickness, both in micrometers. Assume $a=D / 2=1.25 \mathrm{~mm}$ and $h=170 \mu \mathrm{m}$, which are the same parameters as the diaphragm used in the sensor with the through hole; then the air gap change is calculated to be $518.86 \mathrm{~nm}$ when the pressure is increased from 20 to 80 psi, which is 2.28 times the linear operating range of the sensor. In comparison, the through hole in the proposed sensor reduces the pressure-dependent air gap change to $2.31 \%$ of this value, or $12 \mathrm{~nm}$.

\section{Conclusions}

In summary, a new diaphragm-based EFPI sensor structure for acoustic wave detection has been presented. The sensitivity of the sensor to background pressure is significantly reduced, eliminating the need for operating point control procedures in some applications, and thus reducing the system cost. This structure also provides the potential advantage of temperature insensitivity and high-temperature capability if fused silica ferrules and diaphragms and epoxyfree bonding are used.

\section{References}

1. K. A. Murphy, M. F. Gunther, A. Wang, and R. O. Claus, "Detection of acoustic emission location using optical fiber sensors," in Smart Structures and Materials 1994: Smart Sensing, Processing, and Instrumentation, J. S. Sirkis, Ed., Proc. SPIE 2191, 282-290 (1994).

2. J. Deng, H. Xiao, W. Huo, M. Luo, R. May, A. Wang, and Y. Liu, "Optical fiber sensor-based detection of partial discharges in power transformers," Opt. Laser Technol. 33, 305-311 (2001).

3. B. Yu, D. Kim, J. Deng, H. Xiao, and A. Wang, "Fiber Fabry-Perot sensors for detection of partial discharges in power transformers," Appl. Opt. 42, 3242-3250 (2003).

4. N. Furstenau, M. Schmidt, H. Horack, W. Goetze, and W. Schmidt, "Extrinsic Fabry-Perot interferometer vibration and acoustic sensor systems for airport ground traffic monitoring," IEE Proc.: Optoelectron. 144, 134-144 (1997).

5. W. Pulliam, P. Russler, R. Mlcak, K. Murphy, and C. Kozikowski, "Micromachined, SiC fiber optic pressure sensors for high temperature aerospace applications," in Industrial Sensing Systems, A. Wang and E. Udd, Eds., Proc. SPIE 4202, 21-30 (2000).

6. J. Xu, G. R. Pickrell, B. Yu, M. Han, Y. Zhu, X. Wang, K. L. Cooper, and A. Wang, "Epoxy-free high-temperature fiber optic pressure sensors for gas turbine engine applications," Proc. SPIE 5590, 1-10 (2004). 